\title{
tic\&société
}

Vol. 2, $n^{\circ} 1 \mid 2008$

Tic et santé

\section{TIC et santé : entre information médicale et} information de santé

\section{Hélène Romeyer}

\section{Q OpenEdition \\ 1 Journals}

Édition électronique

URL : http://journals.openedition.org/ticetsociete/365

DOI : 10.4000/ticetsociete.365

Éditeur

Association ARTIC

Référence électronique

Hélène Romeyer, «TIC et santé : entre information médicale et information de santé », tic\&société [En ligne], Vol. 2, n 1 | 2008, mis en ligne le 13 octobre 2008, consulté le 19 avril 2019. URL : http:// journals.openedition.org/ticetsociete/365; DOI : 10.4000/ticetsociete.365 


\title{
TIC et santé : entre information médicale et information de santé
}

\author{
Hélène ROMEYER \\ helene.romeyer@u-grenoble3.fr \\ Université de Rennes 1 \\ IUT de Lannion \\ Département Information/communication \\ Rue Edourad Branly \\ BP 30219 \\ 22302 LANNION CEDEX
}

Hélène Romeyer est maître de conférences à l'Institut universitaire de technologie (IUT) de Lannion, Université de Rennes 1 et membre associée au Groupe de recherche sur les enjeux de la communication (GRESEC- EA 608). Ses recherches s'intéressent particulièrement aux problématiques de santé dans les rapports sciences / société. Après un post doctorat à l'Institut National du Cancer, l'auteure continue à travailler sur les phénomènes de publicisation et de médiatisation des thématiques de santé. Par ailleurs, ses travaux de recherche portent sur les pratiques journalistiques et la production multi-supports au sein du Centre de Recherches sur l'Action Politique en Europe (CRAPE- UMR 6051). 


\title{
TIC et santé : entre information médicale et information de santé
}

\begin{abstract}
Résumé
L'objet de cet article est de mettre en évidence que, sous l'effet des technologies d'information et de communication (TIC), deux types distincts d'informations à propos des thématiques de santé émergent : information de santé (grand public, non spécialisée) et information médicale (professionnelle). Gagnant peu à peu les supports de presse, cette distinction est extrêmement prégnante sur l'Internet. Ces deux informations renvoient par ailleurs à de nouveaux usages en matière d'informations sur la santé et à une certaine forme d'industrialisation d'une information jusque-là publique et scientifique, tout du moins en France. Cet article s'appuie sur l'analyse d'un site francophone, Doctissimo (www.doctissimo.fr), et plus exactement de son forum de discussion, ainsi que sur des entretiens semidirectifs menés auprès d'internautes et de médecins.
\end{abstract}

\section{Mots clés}

Information scientifique et publique ; Internet ; information de santé ; information médicale ; industrialisation.

\begin{abstract}
This article attempts to show evidence of the emergence of two distinct kinds of information as a result of information and communication technologies: health information and medical information. While it is gradually gaining credence in the press, this distinction is extremely significant on the Internet. Notably, those two kinds of information refer to new uses of health information, as well as forms of industrialisation of that information that has been until now relatively public and scientific. The article is based on analysis of the discussion forum of a French language site, Doctissimo (www.doctissimo.fr),as well as on semi-structured interviews with Internet users and physicians.
\end{abstract}


TIC et santé : entre information médicale et information de santé

\section{Keywords}

An information public and scientific, Internet, health information, medical information, forms of industrialisation.

\section{Resumen}

El objeto de este artículo es poner en evidencia que bajo el efecto de las tecnologías de información y comunicación, emergen dos tipos distintos de informaciones: información de salud e información médica. Ambas informaciones reenvían entonces a nuevos usos en cuestión de informaciones sobre la salud y a una cierta forma de industrialización de esta información, hasta este momento, pública y científica.

\section{Palabras Clave}

Información pública y científica, Internet, información de salud, información médica, industrialización 
Hélène ROMEYER

\section{Introduction}

Au cours des dernières années, le secteur professionnel de la santé en France a été restructuré par différentes politiques publiques (Carré, 2002, Convert et Demailly, 2003). Si la maîtrise des dépenses est devenue, en France comme ailleurs, le principal objectif poursuivi par les autorités publiques, le déploiement des techniques d'information et de communication (TIC) a provoqué un glissement de l'information médicale à l'information de santé.

En effet, de façon concomitante à cet effort de rationalisation, l'Internet s'est développé et, à la faveur de plusieurs affaires politico-juridiques très médiatisées et d'actions militantes largement publicisées, les thématiques de santé ont gagné l'espace public (Pailliart et Lafon, 2007). Dès lors cette émergence des thématiques de santé, l'action publique de rationalisation et les affaires médiatisées ont multiplié les discours et donc l'information ayant trait à la santé. Ces discours et cette information dépassent désormais le seul cadre médical. Les instances de production et de diffusion ont augmenté au point de rendre l'offre d'information pléthorique. Des laboratoires pharmaceutiques, des mutuelles, des assurances, des associations de malades, des universités, des centres de recherche, les institutions publiques, tous produisent de l'information. Et cette dernière connaît des statuts fort différents : mises à disposition d'informations, comptes rendus scientifiques, stratégies commerciales pour tel ou tel médicament, conseils, informations pratiques, consultation en ligne, archives, services gratuits, services payants, etc. De véritables industries du contenu se sont spécialisées dans le domaine de la santé.

L'objet de cet article est de mettre en évidence l'émergence de deux types distincts d'informations, l'information de santé et l'information médicale, ainsi que les nouveaux usages en matière d'informations sur la santé dont le succès du forum Doctissimo est un cas exemplaire (http://www.doctissimo.fr). On observe conséquemment l'arrivée de nouveaux acteurs notamment du secteur privé et le développement de nouvelles industries de contenu dans le domaine de l'information relative à la santé. Ces mutations révèlent une certaine forme d'industrialisation de l'information relative à la santé.

Plus particulièrement, il s'agit ici de montrer la coexistence de deux types d'information: d'un coté, une information médicale, traditionnellement produite par des spécialistes et répondant aux critères et au fonctionnement de l'information publique scientifique et, de l'autre, une nouvelle catégorie d'information, à destination du grand public, diffusée sur Internet. Cette information que nous appellerons de santé voit sa production échapper aussi bien au secteur médical qu'à l'État. Cette cohabitation créée une tension entre les professionnels de la santé attachés à l'information médicale professionnelle 
TIC et santé : entre information médicale et information de santé

spécialisée, très encadrée et réglementée par l'État, et les industriels ou les entreprises de contenu souhaitant développer une information de santé, à destination du grand public et échappant à tout contrôle. Paradoxalement, alors que plusieurs travaux ne cessent de s'interroger sur la fiabilité ou validité de cette information (Nabarette, 2002, Bourret 2003), l'internaute usager semble, lui, l'avoir intégrée, sait orienter sa recherche et développe un usage fort différencié de ces deux types d'informations (Paganelli et al., 2008).

Cet article s'appuie sur les résultats d'une recherche postdoctorale menée par l'auteure pour le compte de l'Institut national du cancer ${ }^{1}$ et qui cherchait à retracer les discours médiatiques produits sur le cancer. II prend également appui sur divers travaux de recherche menés au sein du Gresec et notamment un groupe de travail «Information et santé » qui développe des méthodologies tant sur le plan informationnel que communicationnel à propos de l'information de santé sur l'Internet. Dans le cadre de ce projet, plusieurs fils de discussion, tout thème confondu, ont été analysés sur la base d'étude lexicographique, d'analyse de contenu et d'analyse cognitive, et, à titre individuel, l'auteure a mené une enquête auprès d'internautes usagers du forum Doctissimo intervenant sur le fil de discussion «cancer». 32 internautes usagers ont ainsi été questionnés. Enfin, dans le cadre d'une étude sur les mutations de l'information publique ${ }^{2}$, l'auteure avait réalisé une série d'entretiens avec des médecins des départements d'information médicale de plusieurs hôpitaux en France. 12 médecins ont ainsi été rencontrés.

II s'agissait, au travers de questionnaires fermés pour les internautes et d'entretiens semi-directifs pour les médecins, de cerner les usages de l'information de santé sur Internet des uns et des autres. Nous avons ainsi cherché à retracer le profil sociologique des usagers de Doctissimo, leur ancienneté sur le forum et leurs motivations. Nous nous sommes attachée à définir quel type d'information ils venaient chercher sur ce forum, comment ils la définissaient et la différence qu'ils faisaient ou pas avec l'information médicale accessible par d'autres moyens.

Les entretiens menés auprès des médecins nous ont permis d'évaluer quelle était l'image de l'information médicale accessible sur l'Internet pour ce corps professionnel et quelle définition ils en donnaient. Après avoir cerné si euxmêmes étaient utilisateurs d'Internet, nous avons questionné les changements

\footnotetext{
${ }^{1}$ Romeyer Hélène, «La publicisation de la lutte contre le cancer en France entre 2000 et 2005 », étude menée dans le cadre du post doctorat à l'Institut national du Cancer, 2008, Rapport non publié.

2 Une partie de cet article a été présenté oralement par Isabelle Pailliart et Hélène Romeyer au colloque international «Mutations des industries de la culture de l'information et de la communication », MSH Paris Nord, 25, 26, 27 Septembre 2006.
} 
induits dans la relation médecin-patient par la présence de patients très informés.

Ce travail de terrain, appuyé sur la lecture des travaux en sciences de l'information et de la communication, mais pas uniquement, nous permet en deux étapes de montrer le passage de l'information médicale à l'information de santé sous l'influence des TIC : tout d'abord la remise en question d'un modèle jusque-là traditionnel, celui de l'information médicale publique et scientifique, puis l'émergence de l'information de santé grand public. Enfin, l'analyse des usages de l'information de santé révèle que, contrairement aux craintes des professionnels, l'internaute usager semble particulièrement faire la différence entre ces deux types d'information.

\section{L'information médicale : un modèle traditionnel mis en difficulté}

De nombreux auteurs ont analysé la réforme du système de santé publique, le "virage ambulatoire ", l'imposition de l'informatique, puis la numérisation des données professionnelles et spécialisées (Carré, Panico, 2000 ; Convert, Demailly, 2003). La volonté de réduire les coûts des dépenses de santé est ainsi apparue à partir des années 1970, période durant laquelle le coût de la santé s'est élevé en France trois fois plus rapidement que le PIB en franc constant (Convert, Demailly, 2003). De nouvelles règles de financement et un système de maîtrise des dépenses sont mis en place en diminuant la part prise en charge par l'État (Carré, Panico, 2000 : 11). Historiquement, les techniques d'information et de communication ont toujours été au service de l'information publique, c'est-à-dire que les gouvernements français successifs se sont appuyés sur les TIC pour diffuser l'information de leurs services et moderniser leurs administrations. Ainsi, après le rapport Nora-Minc de 1978, la télématique (Minitel) est utilisée par l'administration française. Plus tard, c'est Internet qui prendra le relais comme outil de modernisation de cette information. Dans le domaine de la santé, l'État français pourtant habitué à ces outils, n'a pas su ou voulu les mettre en œuvre dans la maîtrise de l'information de santé grand public. II a concentré ses efforts sur l'information médicale professionnelle dans une logique de maîtrise des coûts et de rationalisation de l'organisation du travail : "l'économie de la santé devient progressivement le savoir scientifique à partir duquel est pensé la politique de santé publique" (Serré, 2002 : 79). Se focalisant sur cette seule mission d'administration et de régulation financière, l'État français n'a pas su ou voulu endiguer les mutations liées aux TIC et à leurs usages grand public du point de vue de l'information ayant trait à la santé.

La question de l'information publique comme celle de la communication publique est à replacer dans le contexte plus large des relations entre une institution, son appareil administratif et ses administrés. De ce point de vue, 
TIC et santé : entre information médicale et information de santé

l'évolution française est marquée par le souci de diffuser de l'information sur ses activités et la nécessité d'améliorer et rationaliser ses relations avec les usagers. Dans les années 1970 se diffuse le " principe selon lequel l'information représente un nouveau droit dans le contrat entre l'État et la nation" (OllivierYaniv, in Olivesi, 2006 : 99). Le projet d'information publique s'inscrit dans une stratégie d'ouverture de l'administration aux citoyens. Pour faire face au fossé s'élargissant entre les citoyens et leur administration, on diffuse massivement de l'information. En cela, il s'agit d'une "volonté de réorganisation de l'administration » (Ollivier-Yaniv, in Olivesi, 2006 : 100). Le secteur de la santé n'échappe pas en France comme dans d'autres pays industriels comme le Canada (Grosjean, Bonneville, 2007) à cette réorganisation. Réorganisation dans laquelle les TIC sont investies d'un pouvoir collaboratif et d'une mission de partage des connaissances (Benghozi 2001, Carré, Lacroix, 2001, Bonneville, 2003).

Cependant, devant l'échec d'une maîtrise financière conduite par les médecins eux-mêmes, les pouvoirs publics en viennent à imaginer un contrôle plus strictement comptable. C'est ainsi que l'informatisation et la rationalisation du secteur de la santé deviennent des outils de la réforme dans les années 1990. La chronologie des transformations successives du système de santé laisse entrevoir un mouvement de longue durée portant aussi bien sur les processus de production que sur les articulations production/consommation ou sur les mécanismes de formation des usages. Ainsi, toujours en France, la loi du 4 janvier 1993, dite Loi Teulade, établit le codage des actes et des pathologies diagnostiquées, alors que l'ordonnance d'avril 1996 crée les Agences Régionales de l'Hospitalisation qui fournissent la mise en place des Schémas régionaux d'Organisation Sanitaire (SROS), fer de lance de la politique territoriale intégrée du soin. De plus, depuis 1991, l'informatisation est encadrée par les Programmes de Médicalisation des Systèmes d'Informatisation (PMSI) et un recueil systématique, avec un traitement automatisé de l'information médico-administrative, est mis en place. Ce processus est renforcé depuis 2004 par la carte vitale qui régit l'accès et les modalités d'utilisation du Réseau Social Santé (RSS). Le Dossier Patient Informatisé (DPI) signe de son côté l'avènement du patient virtuel. L'objectif technique réside dans la décentralisation de la saisie des données-patients et, simultanément, dans leur interconnexion possible en tout lieu habilité à le faire. C'est donc bien l'informatisation de données à la fois médicales et gestionnaires. Ces réglementations ne touchent cependant que l'information professionnelle spécialisée, et non l'information grand public. Un premier clivage commence à s'établir ici entre l'information médicale, professionnelle, et l'information de santé, grand public. 
La mise en place de ce système de circulation d'informations provenant d'actes médicaux dénote d'une forme nouvelle d'industrialisation du secteur de la santé. En effet, la numérisation des données médicales, des actes médicaux et de leurs comptes rendus, facilite l'établissement de bases de données et l'étalonnement sur des critères de performance. Toutefois, l'évolution ne saurait se limiter à la modification de l'organisation du travail. C'est en fait le développement de l'usage des technniques d'information et de communication pour et par les professionnels, et pour et par les patients, qui s'installe de façon pérenne. C'est ainsi la seule profession qui s'est vue imposer l'informatisation par le législateur. "Tout cela a amené une certaine rationalisation de l'information médicale par l'introduction de NTIC, pour des objectifs de maîtrise des coûts et d'amélioration des services. Du coup, les pratiques professionnelles comme les mentalités ont évolué ${ }^{3}$ ». La mise en réseau est ainsi l'occasion pour de nouveaux acteurs privés de se positionner favorablement au sein d'un champ d'activités en forte croissance économique et de proposer une relation de plus en plus contractuelle et marchande.

La santé en France a toujours était conçue comme relevant du domaine public, c'est-à-dire qui a trait à l'État. Dès lors, l'information médicale est une information publique, au sens fort du terme. La spécificité des données lui donne sa deuxième caractéristique: elle est scientifique, c'est-à-dire qu'elle répond à des normes de production et de validation par une communauté avant diffusion. En tant qu'information publique, elle s'inscrit pleinement dans les modalités de fonctionnement des politiques publiques; en tant qu'information scientifique, elle doit répondre aux critères précis d'évaluation et de diffusion de la communauté scientifique avant de s'adresser au grand public. Or, actuellement, cette information de santé connaît un mouvement complexe de publicisation qui interroge la signification du caractère public de l'information. En effet, non seulement des éléments touchant éminemment au domaine privé sont publicisés, mais surtout les malades, leurs proches ou des associations produisent de l'information via les TIC sans passer par la sphère médicale ou scientifique. Désormais, en France, la production d'information de santé n'est plus l'apanage du secteur médical, ni du secteur public (État), comme cela était le cas traditionnellement.

En effet, plusieurs mutations sont à l'oeuvre. D'abord, l'information publique médicale est marquée par un mouvement de privatisation. Cette expression comprend l'intervention d'acteurs de la sphère économique: Astra Zeneca, quatrième groupe pharmaceutique mondial développe une base de données multimédia destinée au grand public; Airmedis, opérateur et fournisseur de services wi-fi, équipe les hôpitaux; Medcost lance Doctissimo. $\mathrm{fr}^{4}$, etc. Cela

\footnotetext{
${ }^{3}$ Dr Jehan, entretien réalisé le 5 août 2006.

${ }^{4}$ Racheté depuis par le groupe Lagardère.
} 
TIC et santé : entre information médicale et information de santé

signifie également un mouvement de privatisation par les individus eux-mêmes, avec l'utilisation, dans la sphère privée et pour des objectifs strictement individualisés, de l'information médicale. Ce mouvement de privatisation s'accompagne de formes de marchandisation de l'information publique. Celle-ci devient un enjeu commercial à plus d'un titre. Si les enjeux financiers de l'information médicale ne sont pas nouveaux, ils s'accentuent et revêtent cependant des modalités nouvelles.

Par ailleurs, les supports d'information médicale sont de plus en plus nombreux et leur production échappe désormais au seul secteur scientifique. Avec la multiplication des instances de production et de diffusion, le statut de cette information se diversifie. Les laboratoires pharmaceutiques, les assurances, les associations de malades, les centres de recherche ou les institutions publiques produisent et diffusent désormais de l'information qui dépasse largement le simple cadre de l'information médicale sous formes d'informations pratiques, de conseils, de promotion de tel ou tel médicament, ou de comptes rendus scientifiques, etc.

Enfin, ce secteur connaît lui aussi une remise en cause des paroles d'autorité, ou une extension dans la sphère médicale de comportements militants propres à la sphère politique: développement d'une information "alternative », expressions publicisées des individus, refus des statuts et de l'expertise légitimée uniquement par ceux-ci. Ces usagers de la santé investissent ainsi le forum de Doctissimo pour remettre en cause les légitimités classiques et anciennes du corps médical : "Sur le forum, certains membres m'ont conseillé de changer de médecin, et ils ont bien fait ", ou encore "mon médecin ne voulait pas m'écouter alors j'ai demandé sur le forum ", et enfin " avec Internet, on peut suivre les avancées thérapeutiques et s'inscrire à des protocoles d'essai à l'étranger $»^{5}$.

Le législateur n'a donc pas ou très peu œuvré à propos de l'information de santé grand public, non professionnelle, car elle n'entrait pas dans sa logique de rationalisation des dépenses. Une privatisation partielle de l'information médicale s'est ainsi opérée à partir de la fin des années 1990 sous l'impulsion des TIC. Les opérateurs de cette privatisation partielle, pour des raisons économiques évidentes, se sont tournés vers le grand public faisant ainsi glisser l'information médicale vers l'information de santé.

\footnotetext{
${ }^{5}$ Extraits d'entretiens réalisés entre janvier et mai 2005 auprès d'internautes usagers du fil cancer de Doctissimo ou abonnés à Jeunesse Solidarité Cancer, site dont l'auteure a rencontré le webmestre en entretien lors de son postdoctorat.
} 


\section{L'information de santé sur Internet ou le contournement du modèle traditionnel}

Le mouvement de réforme du système de santé publique correspond chronologiquement au développement des TIC et notamment d'Internet. Le Programme d'Action Gouvernemental pour la Société de l'Information (PAGSI), adopté en 1998, indiquait d'ailleurs clairement que la modernisation des systèmes d'information du secteur sanitaire et social est une priorité dans l'optique de "préparer l'entrée de la France dans la société de l'information " (Carré, Panico, 2000 : 20). Toutefois, rappelons que l'informatisation comprend deux phases essentielles, dans un premier temps, celle de la numérisation de l'information, c'est-à-dire l'avènement de l'ordinateur, puis celle de l'extension de la numérisation au transport de l'information, la communication. Si, dans le domaine de la santé, l'État français a pris en charge et contrôlé la numérisation de l'information, il n'a pas fait le même effort pour le transport de l'information qu'il a en quelque sorte abandonné à des opérateurs privés pour l'information professionnelle et à des industries du contenu pour l'information grand public. « [Or], c'est précisément la numérisation du transport de l'information qui permet de réaliser le potentiel de transformation socio-économique que contient en germe l'informatisation »(Rallet, in Olivesi, 2006 :146).

Le législateur s'est concentré sur l'information professionnelle car son unique objectif était de maîtriser les dépenses de santé, et a négligé ainsi l'information grand public. Des acteurs privés, ayant entrevue le potentiel économique du secteur de la santé en ligne, ont profité de cet oubli pour développer par exemple des sites Internet leur permettant de contourner aisément la loi. D'une part, ne s'intéressant pas à l'information médicale, aux données de santé de patients, ils ne sont pas tenus de respecter le cadre législatif français de l'information médicale, d'autre part, n'étant pas médecins, ils ne sont pas soumis non plus à l'encadrement du Conseil de l'Ordre ${ }^{6}$. Ces sites permettent aussi à certains industriels de se soustraire aux lois sur la publicité ou la vente de médicaments.

Dès lors, le mouvement d'ouverture à des acteurs privés, enclenché par la réforme du système de santé, s'accentue avec le développement de la santé en ligne. Ce qui était jusque-là une prérogative publique et scientifique devient une information non réglementée et non contrôlée. Notons que si l'utilisation des TIC en milieu professionnel et les changements organisationnels qui en découlent

\footnotetext{
${ }^{6}$ Le Conseil de I'Ordre a diffusé à plusieurs reprises des mises en garde quant à l'utilisation d'Internet à travers son bulletin officiel. Par exemple : "Informatique et numérisation : avant tout sécuriser ", septembre 2003 ; "Médecine par Internet ou téléphone : comment éviter les dérives ?", juin 2005 ; «le dossier médical personnel : I'Ordre un partenaire vigilant », mars 2006 ; "Internet et santé : une certification pour l'information de qualité demandée ", janvier 2008. (http:// bulletin.conseil-national.medecin.fr)
} 
TIC et santé : entre information médicale et information de santé

ont été analysés (Dominique Carré pour la France, Luc Bonneville pour le Canada), l'impact des industries du contenu dans le domaine de la santé sur les professionnels et sur les patients ainsi que les répercussions de la spécialisation de l'information sur les organisations de santé, ont été à notre connaissance peu étudiés. C'est pourquoi à travers l'exemple du portail Doctissimo, nous souhaitons réintroduire une réflexion sur le développement des industries de contenu et certaines formes d'industrialisation.

L'histoire de ce portail francophone dédié à la santé est révélatrice de la rentabilité du secteur et de l'engouement dont il est l'objet. L'évolution de ce site montre comment, dans un premier temps, les éditeurs de contenu sont venus développer l'information de santé pour contourner l'encadrement restrictif de l'information médicale, pour, une fois leur entreprise stabilisée, profiter dans un deuxième temps de la possibilité donnée au secteur privé d'intervenir dans les instances publiques. En effet, comme l'a souligné Bernard Miège, « les TIC, au fur et à mesure de leur insertion sociale, sont tout à la fois l'origine et le vecteur d'une industrialisation renforcée des champ sociaux» (Miège, 2000: 73). Finalement, arrivé à maturité, rentable financièrement $(8,65$ millions d'euros de chiffre d'affaires en 2006, 11,47 millions d'euros en 2007) ${ }^{7}$ et classé parmi les sites Internet les plus visités en France (22ème rang des sites les plus visités en France avec 6241000 visiteurs uniques tous lieux de connexion en 2008), Doctissimo devient la cible des grands groupes médiatiques qui lorgnent désormais sur son audience.

Les tendances à l'individualisation et la dématérialisation des supports engendrés par l'utilisation et le développement des TIC ne sont plus à démontrer dans le domaine de la santé comme ailleurs (Carré 1994, Bonneville 2003, Miège, 2000). Par contre, l'élargissement des consommations marchandes semble être actuellement le véritable enjeu dans le domaine de la santé. Et c'est à travers cet enjeu que l'on peut étudier le développement et le succès des sites dédiés à la santé. En effet, les TIC ne provoquent pas d'accroissement quantitatif des marchés existants mais leur élargissement avec de nouvelles activités sociales qui deviennent des cibles commerciales privilégiées ${ }^{8}$. Le succès rencontré par les thématiques de santé dans les grands médias de masse et la prégnance des affaires politico-médiatiques liés à des scandales ou problèmes sanitaires, ont fait de ce secteur une cible toute désignée pour ces nouvelles industries du contenu.

Doctissimo est ainsi créé en mai 2000 par Medcost, société de conception et de maintien technique de sites dans le domaine de la santé dont le docteur

\footnotetext{
${ }^{7}$ Source Doctissimo, reprise par l'Agence Française de Presse (AFP).

8 Processus que Philippe Bouquillion a particulièrement bien mis en évidence pour le spectacle vivant.
} 


\section{Hélène ROMEYER}

Claude Malhuret ${ }^{9}$ est un des fondateurs. Déficitaire depuis sa création, le site atteint l'équilibre financier en 2003. Quatre ans après son lancement, Doctissimo compte environ 2 millions de visiteurs. Si 80000 abonnés étaient comptabilisés après sept mois d'existence, ils étaient, dès 2004, près de 450000 à recevoir la newsletter hebdomadaire. Quant au site, il revendique 6,24 millions de visiteurs uniques par mois ${ }^{10}$. "Une audience qui, depuis six mois, croît au rythme de $10 \%$ par mois en moyenne, précise Cédric Tournay, président du site. La durée des sessions augmente également, elle est de seize minutes environ, contre neuf il y a un an " ${ }^{11}$. C'est donc bien la réforme du système de santé publique qui permet le développement de cette activité autour de l'information de santé. Une information non contrôlée par les professionnels de santé ce qui n'est pas sans poser quelques problèmes. " II est vital que ce soient nous, médecins, qui encadrions cette information grand public. [...] C'est de notre faute. On est resté trop secret, trop longtemps, trop corporatiste. Donc d'autres ont investi la place et se sont chargés de la diffusion de cette information " ${ }^{12}$.

En prospérant, Medcost réussit à interagir avec le secteur public. Ainsi, en 2003, Doctissimo saisit l'opportunité offerte par le projet de loi Douste-Blazy ${ }^{13}$. L'informatisation des données médicales dans les hôpitaux, à travers le dossier médical partagé, nécessite l'intervention d'entreprises privées pour la mise en place technique du projet ce qui incite le gouvernement à faire un appel d'offres. Medcost y répond, entendant profiter ensuite de l'imbrication de ses activités avec celles du site Doctissimo. D'une part, l'entreprise prévoit de créer "Mon Doctissimo", un espace personnel qui assistera le patient dans le suivi de son traitement et, d'autre part, la mise en place du site Doctissimo Pro, dédié aux médecins. II y a donc bien à terme imbrication totale des deux activités, des deux informations, dont une seule est encore réellement publique, au sens fort du terme.

En 2008, Doctissimo est devenu un draineur important à la fois d'internautes et de publicité, étant au 22ème rang pour les sites les plus visités en France, mais au 16ème rang pour les sites constituant les meilleurs supports publicitaires.

Les enjeux de ces mutations sont de deux ordres. Tout d'abord, on observe une marchandisation renforcée, alors que l'élargissement de l'offre d'information, entraîne une consommation différenciée, accentuant ainsi

\footnotetext{
${ }^{9}$ Médecin et homme politique français, Claude Malhuret a été Président et Directeur de Médecins sans Frontières de 1978 à 1986 et Ministre des Droits de l'Homme de 1986 à 1988.

Source Médiamétrie rapport sur le mois de mai 2008 disponible en ligne à .http://www.mediametrie.fr

${ }^{11}$ Interview Journal du Net, 4 avril 2006

12 Dr Erard, entretien réalisé le 10 août 2006.

${ }^{13}$ Cardiologue, ancien ministre de la santé et ancien ministre des Affaires étrangères sous la présidence de Jacques Chirac.
} 
TIC et santé : entre information médicale et information de santé

l'individualisation qui renforce à son tour la diversification (Miège, 2000 ; Bouquillion 2008). Les enjeux économiques sont donc importants. Puis, il y a auto-médiation (Miège, 2000: 100) puisque les TIC et les réseaux favorisent les relations interindividuelles, sans intermédiaires. Cependant, ce discours néolibertaire, porté par les promoteurs d'Internet, véhicule une conception dangereuse du social et des rapports sociaux. Ce qui semble toutefois démenti par l'étude des usagers du site Doctissimo, ceux-ci restant attachés aux formes traditionnelles de l'encadrement médical : le face-à-face, la relation avec leur médecin.

\section{TIC et santé : pour quels usages ?}

Si la mutation est nette pour les usagers, elle demeure plus problématique pour les médecins vraisemblablement pour des questions identitaires. Comme le souligne Jean Caune, la communication sociale ne peut se passer de médiation (Caune, 2000) et les usagers l'ont bien compris. En revanche, les entretiens avec les médecins montrent un véritable malaise vis-à-vis de la coexistence de ces deux informations.

L'enquête, mentionnée en début d'article et réalisée auprès des visiteurs du site Doctissimo, fait apparaître un profil d'internaute âgé de 25 à 45 ans en moyenne, inscrit depuis relativement longtemps: $75 \%$ des enquêtés fréquentent le site depuis plus d'un an, dont $33 \%$ plus de 2 ans. Les succès de Medcost sont particulièrement efficaces dans le référencement du site puisque $98,3 \%$ des internautes interrogés ont abouti sur Doctissimo en passant par un moteur de recherche. Les internautes viennent sans surprise sur Doctissimo parce qu'ils sont malades ou ont un proche malade. Cependant, $75 \%$ déclarent aussi avoir un intérêt pour les questions de santé. Si l'on recoupe les individus déclarant être malades ou avoir un proche malade et ceux s'intéressant aux questions de santé, les chiffres ne se recouvrent pas. II y a donc des individus non-malades qui se connectent à Doctissimo parce qu'ils ont un intérêt pour les questions de santé. Cette déclaration d'intérêt des internautes pour les sujets de santé vient corroborer la bonne santé de la presse magazine de santé, et autres émissions consacrées à ce thème. Intérêt qui fait de ces usagers une cible commerciale préférentielle pour les industries de contenu.

D'après notre enquête, ce que viennent chercher prioritairement les internautes sur ce site, ce sont des conseils pratiques $(91,6 \%)$, des renseignements sur une maladie $(74,8 \%)$, loin devant le contact avec d'autres malades $(25 \%)^{14}$. Les usagers participent effectivement au forum «pour

\footnotetext{
${ }^{14}$ Le total dépasse les $100 \%$ car les individus pouvaient formuler un maximum de deux réponses.
} 


\section{Hélène ROMEYER}

apporter ou rechercher des informations ou un réconfort moral ${ }^{15}$ » La publicité sur les médicaments et les consultations en ligne étant interdites en France, Doctissimo contourne la loi de deux façons. D'abord, on laisse les internautes, sous l'anonymat de leurs pseudonymes, s'exprimer sur le forum et se conseiller mutuellement. Ainsi, Doctissimo ne peut pas être accusé d'exercice illégal de la médecine ou de publicité sur des médicaments. Par ailleurs, cela entretient le discours néo-libertaire propre à Internet dont nous avons parlé précédemment. Deuxième astuce, sans citer des produits précisément, on conseille une gamme élargie en parlant de ses effets plutôt qu'en donnant son nom. C'est ainsi que les forums de ces sites Internet sont tous dotés de modérateurs qui sont également médecins.

Toutefois, l'attitude des internautes vis-à-vis de l'information glanée sur Internet est très paradoxale. Ils n'arrivent pas à trancher sur la différence que pourraient avoir ces informations par rapport à celles fournies par leur médecin : $52 \%$ pensent que les informations sont différentes, $48 \%$ déclarent le contraire, toujours d'après notre enquête. Ils s'accordent pour reconnaître à Internet «un langage généralement plus adapté au profane ", sur le fait que «les personnes atteintes d'une même maladie peuvent mieux se comprendre", qu'elles "peuvent se donner des trucs», «partager leurs expériences». Internet permettrait de « se placer du côté du patient et non du côté de la science ${ }^{16}$.

Grâce à l'existence de très nombreux sites dédiés à la santé ${ }^{17}$, il est indéniable que les internautes sont très informés. «Ces sources, en accès libre sur Internet, originellement à destination des professionnels, ont très rapidement été utilisées par des patients pour leur propre information médicale » (Senis, $2003, .22)$. Lorsqu'on demande aux internautes de Doctissimo s'ils parlent de ces informations avec leur médecin, la réponse est étonnante. En effet, s'ils discutent avec leur médecin, sur la base d'informations trouvées sur Internet, quitte parfois à remettre en cause leur expertise, ils n'avouent pas avoir trouvé ces informations sur Internet. Ils déclarent craindre la réaction hostile du médecin vis-à-vis d'Internet. Pour ceux qui ont engagé la discussion, si le corps médical « comprend le besoin d'informations du malade », il met néanmoins en garde les patients sur la validité de l'information et le choix des sites.

Et, paradoxalement, alors que les internautes déclarent avoir besoin de compléter les informations du médecin par Internet, celui qu'ils reconnaissent comme légitime en matière d'information de santé reste tout de même le

\footnotetext{
15 Paganelli et al. "Web participatif et santé : de nouveaux rapports à l'information ? ", communication au colloque de l'ACFAS « Web participatif : mutation de la communication ? 6 au 8 mai 2008, Québec. À paraître aux Presses de l'université du Québec.

${ }^{16}$ Extraits de réponses des usagers de Doctissimo. Enquête réalisée par l'auteure en juillet et août 2006.

1721830 groupes sur YahooGroups avec le mot clé «medecine », 64688 pour « health »; 1533 pour « santé » en français et 688 pour « médecine » (in Paganelli \& Al., 2008)
}

tic\&société - 2(1), 2008 
TIC et santé : entre information médicale et information de santé

médecin (cités par $91 \%$ des internautes). Les deux autres sources de légitimité citées sont les chercheurs $(50 \%)$ et les laboratoires pharmaceutiques $(25 \%)$. Les médias en général et Internet en particulier ne sont jamais cités. Manifestement, ce n'est pas une information scientifique, professionnelle, vulgarisée que les internautes viennent chercher. Ainsi, sur 40 fils de discussion de Doctissimo étudiés, le type témoignage, sous la forme de questions ou de réponses, représente environ $50 \%$ des échanges alors que le type information scientifique ne représente que $20 \%$ des échanges (Paganelli et al., 2008). Les usagers semblent avoir intégré la différence entre l'information médicale professionnelle, spécialisée et scientifique, diffusée par le corps médical, et l'information de santé grand public.

Nous avons donc bien une nouvelle forme d'usage de l'information de santé qui est en train de s'installer, et qui vient interférer avec l'information médicale proprement dite. Fondamentalement, ce que montre l'étude de Doctissimo c'est un contournement et non une négation du cadre traditionnel, du cadre médical de l'information. Ce que semblent plébisciter les internautes de Doctissimo c'est une information pratique, donnée dans un langage courant : " des profanes qui s'expriment avec leurs propres mots ", la facilité, l'anonymat et la gratuité de cette recherche d'informations. Mais ils continuent à conférer un rôle prépondérant aux médecins et aux scientifiques.

Même s'il y a une remise en cause de l'expertise unique du médecin par le citoyen plus informé sur la maladie et les traitements, il ne faut sans doute pas y voir une « révolution ». II s'agit plutôt d'un ajustement, d'une revendication de partage de l'expertise bien plus qu'une suppression du cadre traditionnel. Ici c'est le vécu de l'individu, son témoignage, qui est un gage de crédibilité des informations.

De leur côté, les médecins expriment un véritable malaise sur l'existence d'une information de santé qu'ils ne maîtrisent pas. L'information en matière de santé est " marquée par une polysémie certaine ${ }^{18}$. En effet, le terme recouvre à la fois une information plus ou moins commerciale, pharmaceutique auprès des prescripteurs, une information à destination des professionnels de santé via des périodiques professionnels plus ou moins scientifique, une information du grand public sur des problèmes médicaux, et enfin une information médicale au sens de données de santé personnelles.

Les professionnels de santé distinguent l'information médicale professionnelle, technique et scientifique, de l'information de santé vulgarisée à destination du grand public. Ainsi, pour le Dr Alloy, médecin responsable d'un département d'information médicale, "l'information médicale concerne des

\footnotetext{
${ }^{18}$ Entretien avec le Dr Fiegel, réalisé le 8 août 2006.
} 


\section{Hélène ROMEYER}

données de santé pour un individu ou une population, en général issues d'un professionnel de santé. Ses caractéristiques sont la confidentialité, et des règles précises concernant leur conservation, leur utilisation et leur communication "; à différencier, "d'une information de santé qui peut être des données générales : vaccination, diététique par exemple en dehors du professionnel de santé ${ }^{19}$. Pour ces professionnels, ce sont deux mondes différents, qui ne peuvent pas se prévaloir de la même légitimité : "l'information de santé, c'est quelque chose de beaucoup plus vulgarisée que notre monde à nous médecins, extrêmement technique. L'information de santé, c'est de l'information grand public, mais pas expliquée, des bons conseils, des sites Internet qui se consacrent au bien-être ou peut-être la publicité des laboratoires pharmaceutiques ${ }^{20}$. Pourtant les médecins reconnaissent que ces deux informations sont étroitement liées: "L'information médicale qui serait celle utilisée et échangée par les professionnels fait partie de l'information de santé qui est, elle, une catégorie plus large ». D'ailleurs ils déplorent que certains d'entre eux servent de caution à des sites d'information de santé, " l'information de santé est un champ à part, elle n'hésite pas à demander la caution de la médecine ${ }^{21}$.

\section{Conclusion}

Nous avons vu que plusieurs stratégies de contournement du modèle traditionnel d'information médicale publique et scientifique se mettent en place et qu'une nouvelle conception de l'information se dégage de l'usage des informations ayant trait à la santé sur Internet. Le glissement vers un autre modèle est significatif à travers :

- des opérateurs privés qui profitent du relatif vide juridique laissé par l'État français sur l'information de santé grand public, et des possibilités offertes par les technologies d'information et de communication ;

- des malades qui contournent le monde médical par le biais de l'information de santé en ligne, en remettant en cause au passage le face-à-face médecin-patient.

- des médecins et des industries pharmaceutiques qui cautionnent l'information de santé en ligne en faisant partie des équipes scientifiques de ces sites.

Par ailleurs, l'étude des usages des internautes de Doctissimo montre qu'ils ne sont pas en recherche d'une information scientifique, ni d'une explication. Ils

\footnotetext{
${ }^{19}$ Dr Alloy, entretien réalisé le 2 août 2006.

${ }^{20} \mathrm{Dr}$ Erard, entretien réalisé le 10 août 2006.

${ }^{21} \mathrm{Dr}$ Jehan, entretien réalisé le 5 août 2006.

tic\&société - 2(1), 2008
} 
TIC et santé : entre information médicale et information de santé

viennent se rassurer, trouver des gens qui souffrent de la même pathologie qu'eux, discuter avec "ceux qui les comprennent le mieux et vivent la même chose ». De facto, les critiques des médecins sur la véracité et le manque de sérieux des informations médicales sur Internet, et leur potentiel danger d'utilisation, ne trouvent que peu de prise chez les usagers. Cette information grand public et dérégulée constitue un modèle alternatif : l'information de santé. Ce développement de l'information de santé sur Internet pose ainsi plus de problèmes aux médecins pour des questions identitaires et de défense de leur territoire, qu'aux usagers qui font la part des choses, savent saisir les opportunités qui leur sont offertes, mais qui ne sont pas pour autant naïfs en ce qui concerne la valeur de l'information glanée.

Finalement, c'est bien le statut de l'information publique qui est posé à travers ces mutations de l'offre comme des usages. Définie d'abord par son origine, soit l'État et les services publics, elle tend désormais à revêtir un autre statut plus lié à ses pratiques qu'à l'engagement des institutions publiques. L'information publique est alors assimilée à celle qui est mise sur la scène publique et qui sert l'intérêt du plus grand nombre. La "garantie » n'est donc plus fournie par l'autorité de la source mais par la pertinence de ses usages. Devenue plus accessible du fait de la multiplication des acteurs et des registres utilisés, et par conséquent plus éclatée, l'information publique s'intègre dans des logiques socio-économiques comme dans des pratiques de contestation du pouvoir médical.

\section{Références bibliographiques}

BENGHOZI P.-J., 2001, « Technologies de l'information et organisation. De la tentation de la flexibilité à la centralisation », Gestion 2000, vol.2., 2, marsavril, p.61 à 80 .

BONNEVILLE L., 2003, La mise en place du virage ambulatoire informatisé comme solution à la crise de productivité du système sociosanitaire au Québec (1975-2000), Thèse de doctorat en sociologie, université du Québec, Montréal.

BOUQUILLION P., 2008, Les industries de la culture et de la communication. Les stratégies du capitalisme, Grenoble, PUG.

BOUQUILLION P., 1999, « Internet comme vecteur d'industrialisation de la culture : le cas du spectacle vivant », ICUST, second colloque international sur les usages et les services des télécommunications, Bordeaux, Actes, pp.236-245 


\section{Hélène ROMEYER}

BOURRET C., 2003, « la santé en réseaux », revue Études, Vol. 3, Tome 399.

CARRE D., 2002, "Régulation sociale de la diffusion des TIC », mémoire d'Habilitation à Diriger des Recherches, Université Stendhal, Grenoble3.

CARRE D., LACROIX J.-G.(dir), 2001, La santé et les autoroutes de l'information. La greffe informatique, Paris, l'Harmattan.

CARRE D. et PANICO R., 2000, « Les enjeux socio-médicaux de la modernisation de la santé », Terminal, n ${ }^{\circ} 82$, p.7 à 30.

CARRE D., 1994, "Rapports sociaux, médiations technico-culturelles, et évolution de l'espace public : le cas du changement technique en entreprise ", Thèse de troisième cycle, Université Stendhal, Grenoble 3.

CAUNE J., 2000, « La médiation culturelle : une construction du lien social », revue électronique Les Enjeux de l'information et de la communication, http://www.u-grenoble3.fr/les_enjeux/2000/Caune/index.php

CONVERT B. et DEMAILLY L., 2003, « Le savoir médical et sa mise en scène sur le net », Terminal, n ${ }^{\circ} 89$, p. 65 à 76.

CONVERT B.et DEMAILLY L., 2003, «Internet et les professions de santé : le problème de la consultation à distance ", Réseaux n 0120, CNET, Paris.

GROSJEAN S., BONNEVILLE L., 2007, « Logiques d'implantation des TIC dans le secteur de la santé », Revue française de gestion, Vol.3, n¹72, p. 145 à 158.

MIEGE B. 2000, Les industries du contenu face à l'ordre informationnel, Grenoble, PUG.

NABARETTE H., 2002, "L'internet médical et la consommation d'information par les patients ", Réseaux n¹14, Paris, CNET, p.249 à 286.

OLLIVER-YANIV C., 2006, «La communication publique. Communication d'intérêt général et exercice du pouvoir ", dans Olivesi S. (dir de), Sciences de l'information et de la communication. Objets, savoirs, disciplines, Grenoble, PUG.

OLIVESI S. (dir de), 2006, Sciences de l'information et de la communication. Objets, savoirs, disciplines, Grenoble, PUG.

PAGANELLI C., CLAVIER V., MANES-GALLO M.C., MOUNIER E., ROMEYER H., STAll A., 2008, « Web participatif et santé : de nouveaux rapports à l'information ? ", dans Actes du colloque Web participatif : mutation de la communication ?, 6 au 8 mai 2008,Québec (Canada),Presses de l'université du Québec, à paraître. 
TIC et santé : entre information médicale et information de santé

PAILLIART I., LAFON B., 2007, « Malades et maladie dans l'espace public », Questions de communication $n^{\circ} 11$, Nancy, Presses universitaires de Nancy.

PAILLIART I., TAROZZI S., MIEGE B., 1997, «Les premiers pas de la communication mobile professionnelle : le cas de la santé ", rapport de recherche non publié GRESEC.

RALLET A., 2006, « Technologies de l'information et de la communication. Enjeux de société », dans Olivesi S.(dir de), Sciences de l'information et de la communication. Objets, savoirs, disciplines, Grenoble, PUG.

ROMEYER H., 2008, "La publicisation de la lutte contre le cancer en France entre 2000 et 2005 ", étude menée dans le cadre du post doctorat à l'Institut national du Cancer, Rapport non publié.

SENIS F., 2003, "Pourquoi accéder à l'information médicale sur Internet par le biais des groupes de discussions ? Qualité, centres d'intérêt et motivations des participants aux forums médicaux. A propos du forum Usenet Fr.bio.medecine », Thèse de doctorat de médecine générale, Université Bordeaux 2 - Faculté de Médecine [Document en ligne]

http://www-fbm.medinet.fr.eu.org/these/these.pdf

SERRE M., 2002, «De l'économie médicale à l'économie de la santé ", Actes de la Recherche en sciences sociales, vol.143, n¹, Paris, EHESS, p.68-79.

\section{Pour citer cet article}

Hélène Romeyer, «TIC et santé : entre information médicale et information de santé», Revue tic\&société [En ligne], Numéros, Tic et santé, mis à jour le : 13/10/2008, URL :

http://revues.mshparisnord.org/lodel/ticsociete/index.php?id=365. 